\title{
ACCURACY OF TWO DIFFERENT IMPRESSION MATERIALS IN PARALLEL AND NON PARALLEL IMPLANT POSITIONS
}

\author{
Ahmed M. Alam-Eldein* and Enas A. Elshenawy **
}

\begin{abstract}
Purpose: Accurate implant impressions play a significant role and serve as a starting point in the process of producing good working casts. This study was designed to evaluate the effect of two different impression materials, additional silicone (Enthus) and vinyl siloxanether (EXA'lence), on the accuracy of impressions in parallel and nonparallel implants.

Material and Methods: In this study, two completely edentulous mandibular models were used. Three implants (OsseoLink USA LLC. $4 \mathrm{~mm} \times 9 \mathrm{~mm}$, internal connection type) were placed in each reference model with different angles ( 0 and 15 degrees). Thirty stone casts were made from each reference model using additional silicone (Enthus polyvinyl siloxane, PVS) and vinyl siloxanether (EXA'lence, VSXE) ( $\mathrm{n}=15$ per group) with direct technique. The inter-implant distances were measured for casts using a coordinate measuring machine and the deviations compared to the reference models were calculated. Data were collected, tabulated and statistically analyzed using Student T-test.
\end{abstract}

Results: In parallel condition, the two materials showed non-significant difference in deformation of ( $\Delta \mathrm{r} 1$ or $\Delta \mathrm{r} 2)$, while in the presence of angulated implants, vinyl siloxanether (EXA'lence, VSXE) impression material showed more accurate results compared to additional silicone (Enthus polyvinyl siloxane, PVS) impression material.

Conclusion: Within the limitations of this study, in parallel conditions, the type of impression material cannot affect the accuracy of the implant impressions; however, in nonparallel conditions, vinyl siloxanether (EXA'lence, VSXE) impression material produced more accurate casts than additional silicone (Enthus polyvinyl siloxane, PVS) impression material.

KEYWORDS: Dental implant, Polyvinyl siloxane, Vinyl siloxanether.

* Lecturer, Prosthodontic department, Faculty of dentistry, Tanta University,

** Demonstrator, Dental Biomaterials department, Faculty of dentistry, Tanta University. 


\section{INTRODUCTION}

Osseointegrated implants were used for rehabilitation of edentulous patients with the principle objective of replacing conventional complete dentures with an implant supported prosthesis ${ }^{1}$.

Reproducing the intraoral relationship of implants through impression procedures is the first step in achieving an accurate, passively fitting prosthesis $^{2}$. Failing to achieve this passive fit will incur stress on implants, which can finally lead to fracture of the implant components and failure of the treatment ${ }^{3}$.

Several studies have investigated the clinical factors affecting the accuracy of implant impressions, such as direct (open tray) or indirect (closed tray) impression techniques ${ }^{4}$, different impression materials ${ }^{5}$, necessity and method of splinting the impression copings ${ }^{6,7}$, implant angulation ${ }^{8}$, and prosthetic connection features ${ }^{8-11}$.

To date, several implant impression techniques have been introduced and evaluated for accuracy. Two basic impression techniques are commonly used in implant dentistry: the direct (pick-up, open tray) technique and the indirect (transfer, closed tray) technique ${ }^{12}$.

In the direct technique, the top of the impression coping screw is exposed and accessible. The impression copings are unscrewed to be retrieved along with the impression tray once the impression set. The open tray technique allows for the impression coping remaining in the impression. This reduces the effect of the implant angulation, the deformation of the impression material upon recovery from the mouth, and removes the concern for replacing the coping back into its respective space in the impression ${ }^{12}$.

In the indirect technique, the impression copings are connected to the implants before the impression. Closed trays are used. In contrast to the direct technique, the impression copings stay connected to the implant once the impression tray is retrieved, after then these copings will be unscrewed from the mouth and connected to the implant replica. This coping-implant replica assembly will be repositioned into its respective position within the impression ${ }^{4,12}$.

A lack of parallelism among the implants, and that between the implants and the teeth is a common finding in clinic, which is due to anatomical limitations or the esthetic considerations. While unfavorable angulation can be corrected with the restoration, the lack of parallelism in implants and the presence of undercuts create an undesirable path of placement that may distort the impression material upon removal and may produce an inaccurate master cast, especially when multiple implants are used ${ }^{13}$.

Among the impression materials so far used for implant impression, polyether and additional silicone (A-silicon) are mostly suggested $\mathrm{d}^{2,3,8,10,14}$. Sorrentino and colleagues ${ }^{10}$ reported a higher accuracy for addition silicone in the presence of nonparallel implants, whereas polyether achieved the best results with parallel implants and standard impression copings.

Vinyl siloxanether, composed of polyether and additional PVS, is a new impression material, which is claimed ${ }^{15}$ to have all the theoretical advantages of polyether ${ }^{16}$ and PVS such as excellent flowability, remarkable hydrophilicity, easy handling and optimized elastomeric properties. In addition to achieving its high final hardness immediately after the setting, vinyl siloxanether guarantees the precision of the impression. Furthermore, although it has different consistencies (heavy, medium, medium soft and light), the medium one is recommended for implant impression ${ }^{17}$.

Studies comparing the accuracy of implant impression techniques with methods such as micrometers, Vernier calipers, strain gauges, or measuring microscopes could merely carry out two-dimensional measurements. However, when the measurements are 2 dimensional only, relevant information is lost. Therefore, Coordinate measuring 
machine (CMM) was used as the measuring device in this study because it made three-dimensional evaluation of any distortion possible $e^{5,14,18,19}$.

The aim of this study was to evaluate the effect of two different impression materials (Polyvinyl siloxane and Vinyl siloxanether) on the accuracy of impressions in parallel and nonparallel implant positions.

\section{MATERIALS AND METHOD}

\section{Master models fabrication}

Two epoxy resin (Ramses medical products factory, Alex, Egypt) completely edentulous mandibular models representing a clinical situation were used as definitive casts.

Each cast had three implants (OsseoLink USA LLC. $4 \mathrm{~mm} \times 9 \mathrm{~mm}$, internal connection type) arranged with one implant at the midline and the other two implants at the premolar regions.

Cast 1: had three implants parallel to each other and perpendicular to the horizontal plane of the cast.

Cast 2: had implant at the midline perpendicular to the horizontal plane of the cast and two implants at the premolar regions angulated at 15 degree to a line drawn perpendicular to the occlusal plane.
Each master cast was held in a vertical milling machine (Milling \&Drilling machine, RF-Sakkary, Taiwan), and holes matching the depth, diameter and angulation of the implants were prepared.

A protractor was used to align the cutting bur in the proper angulation by tilting the milling machine table (figure 1).

\section{Custom tray fabrication}

\section{Preparation of stone duplicate}

After the impression copings were connected to the master models, the space for impression material was created with two sheets of baseplate wax (Cavex setup waxes, Haarlem, Holland) around the copings and over the ridge area and stoppers ( $2 \times 4 \mathrm{~mm})$ were made on the molar regions to standardize the tray position and impression material thickness at $3 \mathrm{~mm}$.

An impression was taken from each model, using condensation silicone (Zetaplus, Zhermack SpA, Italy). Impressions were boxed and poured with type IV dental stone (elite ${ }^{\circledR}$ stone, Zhermack $\mathrm{GmbH}$ Deutschland) in a vacuum device. The stone was set for 30 minutes and the impression was separated. The two stone casts were used to fabricate all the custom trays.

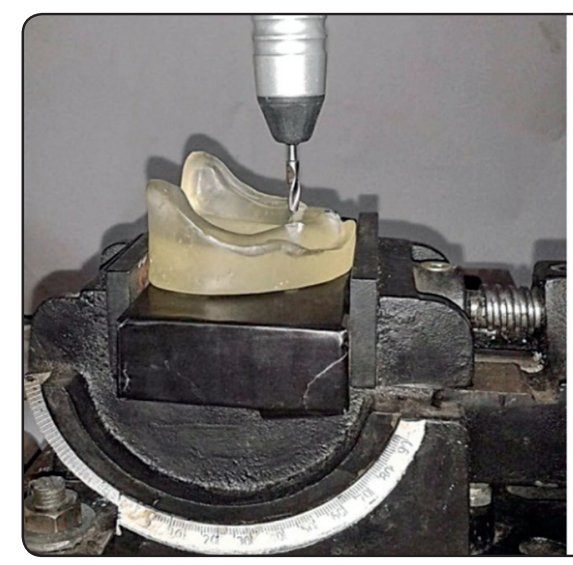

Cast (1)

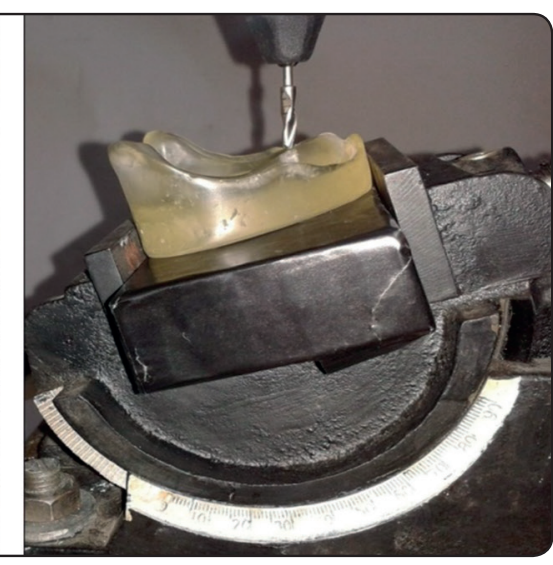

Cast (2)

Fig. (1) The drill at the premolar region is $0^{\circ}$ in cast (1) and $15^{\circ}$ to a line drawn perpendicular to the occlusal plane in cast (2). 


\section{Preparation of the master custom tray}

Separating medium was painted on each stone duplicate before making the master custom trays. Self-cured acrylic resin (Acrostone cold cure special tray material, Cairo, Egypt) was mixed following the manufacturer's instructions and when reached the dough stage, the mix was pressed between two glass slabs to give 2 to $3 \mathrm{~mm}$ thick layer, then it was adapted over the stone duplicate.

Each tray was left for 24 hours to allow for polymerization shrinkage, and then it was removed from the model, trimmed and smoothened. Then replaced on the models and verified for clearance of 2-3 $\mathrm{mm}$ between it and the model.

\section{Preparation of the custom trays:}

A two-part mold was fabricated using each master custom tray and type IV dental stone in a dental flask to make 30 identical custom trays for each cast.

Thirty custom trays were made of a 2-mm selfcured acrylic resin (Acrostone cold cure special tray material, Cairo, Egypt) in the same mold. The trays were trimmed, perforated for added retention of the impression material and windows cut over the implants for direct technique. The trays were stored at the room temperature for $24 \mathrm{~h}$ before impression taking.

\section{Impression Procedure:}

In this study, there were four experimental groups (due to the two impression materials evaluated for two master models) using direct impression technique. A sample size of 15 was used in the experimental groups.

Addition silicon (Enthus PVS Impression Material, Dharma Research, USA) and vinyl siloxanether (EXA'lence, VSXE) impression materials, both with medium consistency, were selected for this study.
The impression protocol was standardized as follows:

1. A $1.5 \mathrm{~kg}$ metal block exerted a standardized pressure on each tray during the polymerization to force the excess material to flow out and to maintain constant pressure throughout the working time.

2. The impression copings were secured with flat head screw on the implant analogues using dedicated torque wrench calibrated at $10 \mathrm{Ncm}$.

3 . The custom trays were filled with regular body impression material mixed using an impression gun. The same material was also syringed around the impression copings on the epoxy resin cast.

After hardening of the impression material, impression copings were unscrewed and all the impressions were removed in the right angle to occlusal plane and checked for accuracy and for the presence of impression copings within impressions. If any inaccuracy, such as bubble, drag, or nonhomogeneous mix of materials was detected, the impression would be repeated. Then, implant analogues were screwed to the impression copings in the impression.

The impressions were stored at room temperature for at least two hours before pouring the casts ${ }^{3}$.

\section{Cast Production Procedure:}

All the impressions were poured with type IV dental stone (elite ${ }^{\circledR}$ stone, Zhermack $\mathrm{GmbH}$ Deutschland) using a single prefabricated mold made with laboratory silicone (Ramses medical products factory, Alex, Egypt) to obtain standardization of the resulting casts and the amount of dental stone used (figure 2).

After the stone had been allowed to set for 1 hour, the casts were separated from the impressions, trimmed, and the three healing abutments were tightened to their respective implant analogues for each cast before the measuring procedures. All casts were labeled and stored at room temperature for a minimum of $24 \mathrm{~h}$ prior to measurements ${ }^{20}$. 


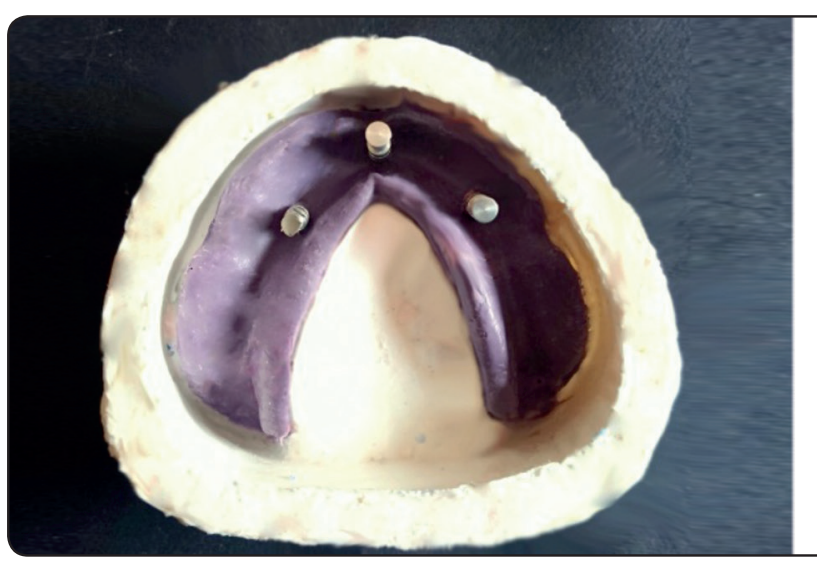

PVS

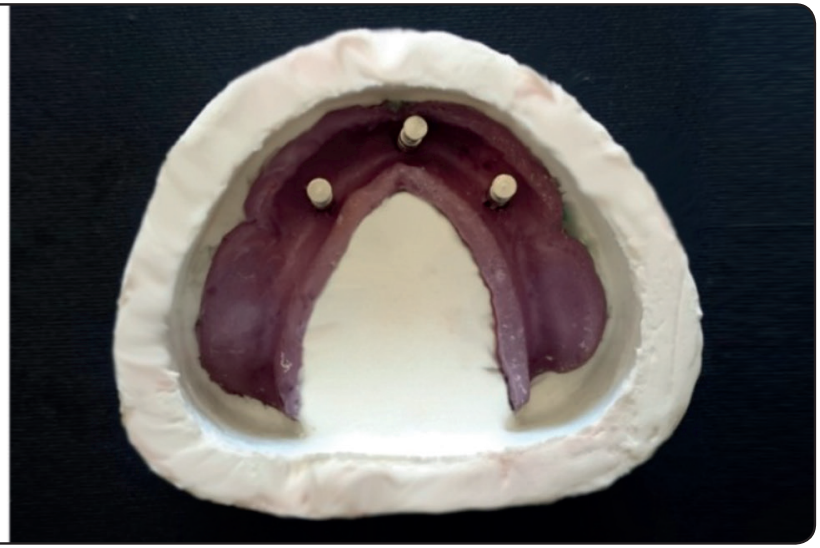

VSXE

Fig. (2) Silicone mold with an impression with the analogue connecting with the coping ready for pouring.

\section{Measurement Procedure:}

A coordinate measuring machine $(\mathrm{CMM})$ (Mitutoyo CRYSTA-Apex S544, Japan) (EgyptJapan University of Science and Technology, Egypt) was used to evaluate the positional accuracy of the samples. The accuracy of the CMM according to the manufacturer was $0.0001 \mathrm{~mm}$. The implant abutments are donated as seen in (figure 3 ).

The center of abutment 1 is considered as the reference point for all measurements. The planar surface from this point was regarded as XY. Two imaginary $\mathrm{XZ}$ lines were considered between the centers of the analogue 1,2 and 1,3. The XZ planes were perpendicular to $\mathrm{XY}$ plane. Therefore, the center of analogue 1 was laid on the origin $(0,0,0)$.

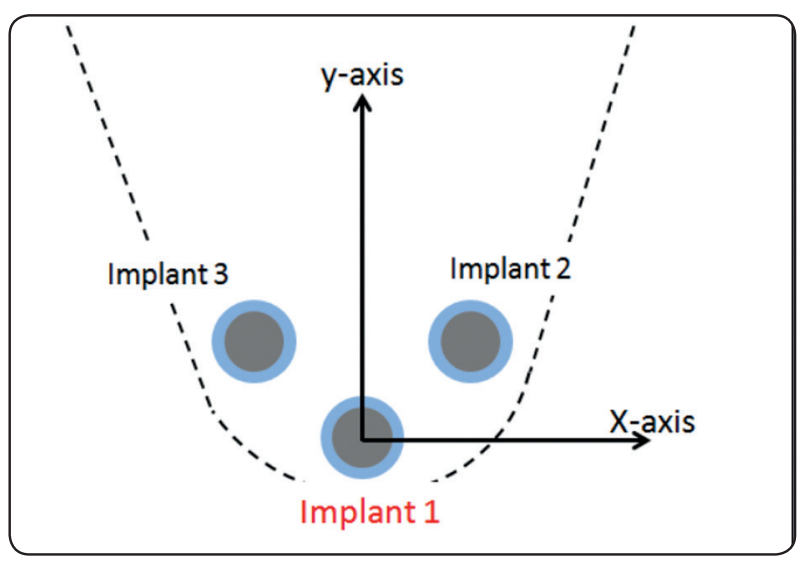

Fig. (3) Implant donations.
For each analogue in the master models as well as the definitive casts, CMM measured the coordinates (X, Y and $\mathrm{Z}$ ) of each analogue with respect to the determined reference axis.

The center of each implant abutments was located using a CMM probe with a diameter of 1 $\mathrm{mm}$ by touching eight points on the circumference of the outer diameter of the implant abutments.

Four points on the upper surface of each implant abutment were measured to form a plane used to calculate the vertical distances between implant abutments 1 and 2 and 1 and 3 in the z-axis.

Three measurements were recorded for inter implant distances in $\mathrm{x}, \mathrm{y}$, and $\mathrm{z}$ axis, and then the mean values were calculated. Six inter implant distances were measured for the reference models and for each of the 60 casts.

The distances (in micrometers) between the implant analog centers with the reference point were calculated according to the following formula ${ }^{21}$ :

The distance from the reference $=\sqrt{\chi^{2}+\mathrm{y}^{2}+\mathrm{z} 2}$.

The error of impression for each pair of implant analogs was computed as the Euclidean distance between the analogs in the duplicated cast with the distance in the master cast regardless of its direction (as the absolute value): 
Absolute error $=$

$\sqrt{\chi 2 m+y 2 m+z 2 m} \cdot-\sqrt{\chi 2 d+y 2 d+z 2 d}$

Where $\mathrm{m}=$ master; $\mathrm{d}=$ duplicated.

The mean average values obtained from the casts were compared with the standard values acquired from the reference model and the differences were calculated. A spreadsheet (Excel 2013, Microsoft) was customized and employed to accomplish this task.

Each model has two Euclidean distances and named $\Delta \mathrm{r} 1$ (between implant abutments 1 and 2) and $\Delta \mathrm{r} 2$ (between implant abutments 1 and 3 ).

\section{Statistical analysis}

Data analysis was performed for each group and descriptive statistics including mean and standard deviation were calculated and reported for each Euclidean distance. Data were statistically analyzed using Student T-test. P values $\leq 0.05$ were considered statistically significant.

\section{RESULTS}

The mean descriptive values of distortion by both impression materials for cast (1) and cast (2) were obtained and provided in table $\mathbf{1}$ and figure 4 .

Student t-test between the two impression material groups in cast (1) indicated no significant difference in deformation of $(\Delta \mathrm{r} 1$ or $\Delta \mathrm{r} 2)$ between PVS and VSXE (P. value 0.39 and 0.11 for $\Delta \mathrm{r} 1$ and $\Delta \mathrm{r} 2$ respectively) as shown in table 2.

TABLE (1) Descriptive mean analysis of test impression materials $(\mu \mathrm{m})$.

\begin{tabular}{|c|c|c|c|c|}
\hline \multirow{2}{*}{ Groups } & \multicolumn{2}{|c|}{ PVS } & VSXE \\
\hline Subgroups & Cast 1 & Cast 2 & Cast 1 & Cast 2 \\
\hline & Mean \pm SD & Mean \pm SD & Mean \pm SD \\
\hline $\boldsymbol{\Delta} \mathbf{r 1}$ & $40.95 \pm 7.7$ & $47.12 \pm 5.4$ & $38.93 \pm 4.5$ & $42.92 \pm 3.3$ \\
\hline $\boldsymbol{\Delta} \mathbf{r} \mathbf{2}$ & $49.01 \pm 7.7$ & $53.14 \pm 6$ & $44.35 \pm 7.6$ & $46.56 \pm 4.1$ \\
\hline
\end{tabular}

$\Delta \mathrm{r} 1:$ the Euclidean distance between implant abutments 1 and 2, $\Delta \mathrm{r}$ : the Euclidean distance between implant abutments 1 and 3.



Fig. (4) Bar graph showing comparison of PVS and VSXE in cast 1 and cast 2
TABLE (2) Showing no significant difference in deformation of ( $\Delta \mathrm{r} 1$ or $\Delta \mathrm{r} 2)$ between PVS and VSXE in cast (1)

\begin{tabular}{|l|l|l|}
\hline & PVS vs VSXE & PVS vs VSXE \\
\hline & $\Delta \mathbf{r} 1$ & $\Delta \mathbf{r}$ \\
\hline T. test & 0.87 & 1.65 \\
\hline P. value & 0.39 & 0.11 \\
\hline
\end{tabular}

Student t-test between the two impression material groups in cast (2) indicated significant difference in deformation of ( $\Delta \mathrm{r} 1$ or $\Delta \mathrm{r} 2)$ between PVS and VSXE (P. value 0.018 and 0.002 for $\Delta \mathrm{r} 1$ and $\Delta \mathrm{r} 2$ respectively) as shown in table 3 . 
TABLE (3) Showing significant difference in deformation of ( $\Delta \mathrm{r} 1$ or $\Delta \mathrm{r} 2)$ between PVS and VSXE in cast (2)

\begin{tabular}{|l|l|l|}
\hline & PVS vs VSXE & PVS vs VSXE \\
\hline & $\Delta \mathbf{r} 1$ & $\Delta \mathbf{r} 2$ \\
\hline T. test & 2.54 & 3.48 \\
\hline P. value & $0.018^{*}$ & $0.002^{*}$ \\
\hline
\end{tabular}

\section{DISCUSSION}

Precise working casts are essential to fabricate passively fitting implant prostheses, regardless of the mode of fabrication. The application of undue torque to screws during attachment of the superstructure to the abutments can jeopardize the outcome. To eliminate discrepancies in fit, it is essential that work should be done on a master cast that reproduces, as accurately as possible, the position of the abutments in the patient's mouth. An important factor that influences the precision of fit is impression accuracy 22,23 .

Three elastomeric impression materials have been used for implant impressions including addition silicon (Enthus polyvinyl siloxane, PVS), poly ether (PE) and vinyl siloxanether (EXA'lence, VSXE) impression materials. PE and PVS have frequently been used for in vitro studies on implant impressions ${ }^{10,19,23}$. Very few studies are available demonstrating the efficacy of VSXE ${ }^{15,24,25}$.

In this study, monophase technique was used as it is accomplished in a single- step procedure using materials with a medium viscosity to allow the material itself to record finer details while avoiding the slumping of the material in the tray, less timeconsuming, and simple to perform ${ }^{26,27}$.

Direct impression technique was selected in this study because research suggests that a direct technique should be used with multiple angulated implants to decrease the distortion ${ }^{8}$.
Two clinical situations were evaluated parallel condition and non-parallel condition with the two implants in the premolar region angulated at 15 degree. This was a simulation to a common clinical situation that may necessitate placement of angulated implants in lower premolar region as the submandibular fossa mandates implant placement with increasing angulation as it progresses distally ${ }^{28}$.

The methodology was standardized to allow a careful evaluation of different procedures, isolating variables associated with laboratory processes. This standardization included the use of custom tray, use of prefabricated mold to pour all casts, impression coping, and a reliable measurement method.

Non-significant difference in deformation of ( $\Delta \mathrm{r} 1$ or $\Delta \mathrm{r} 2$ ) was found between the two materials in parallel condition and this was in agreement with Vojdani et al $^{13}$ while in non-parallel condition, VSXE group produced casts with less distortion values than casts produced by PVS group and significant difference in accuracy between the two tested groups was found and this was in agreement with Shankar et al ${ }^{25}$.

This might be explained by the fact that VSXE is more rigid than regular body PVS, thus preventing movement of the impression copings inside the impression material ${ }^{5}$. Integrating the qualities of PE and PVS into a newer material VSXE has demonstrated good mechanical and flow properties along with excellent wetting characteristics in the unset as well as set conditions. One of the other reasons for improved accuracy of VSXE is the enhancement of the hydrophilicity which may influence the accuracy of impressions and can result in improved flow and finer detail of impressions made on moist dentinal surfaces and in the area of the gingival sulcus ${ }^{24}$. The composition of this new material is intended to incorporate the natural hydrophilicity of conventional PE materials along with the desirable properties of additional silicone materials such as elastic recovery and tear resistance. 
Very few studies have been available in literature citing the accuracy of VSXE. The results of the present study positively supplement the existing studies ${ }^{15,27}$.

If multiple implants are parallel to each other, there will be no horizontal shift in the transfer; if the implants are positioned angled, the rotational misfit leads to a horizontal discrepancy ${ }^{29}$.

It is reported that angular positional transfer deformation increases with an increase in an implant's buccal/lingual inclination with respect to horizontal crestal plane. As implant angulations increase, distortion in the experimental cast increases. This can be explained with increased material deformation upon impression removal. Especially in multiple implant cases, an increase in implant angulation increases the area of friction and the amount of stresses generated in an impression decreasing impression accuracy ${ }^{30}$.

This in vitro study has some limitations. All impressions were taken under ideal conditions without the presence of soft tissues, blood, saliva and sulcular fluid, which may affect the accuracy of the impressions. In addition, the results are limited to three internal connection implants and may not be relevant with higher number of implants and different connection geometries.

\section{CONCLUSIONS}

Within the limitations of the study, it can be concluded that:

1. In parallel conditions, the type of impression material cannot affect the accuracy of the implant impressions; however, in nonparallel conditions, vinyl siloxanether impression material showed lesser deviation from the reference model compared to polyvinyl siloxane impression material.

2. The relation between the angulation and impression material can be established that as with increase in angulation, the amount of forces of deformation increases which require an impression material which can withstand these forces that affect the accuracy of master cast.

3. Further clinical studies testing more implants, different angulations and connection geometry are needed to evaluate the accuracy of implant impressions.

\section{Conflict of interest:}

The Authors declare that they have no conflict of interest, have full control of all primary data.

\section{REFERENCES}

1. Assunção WG, Cardoso A, Gomes EA, Tabata LF, dos Santos PH. Accuracy of impression techniques for implants. Part 1 - influence of transfer copings surface abrasion. J Prosthet Dent. 2008; 17: 641-7.

2. Wee AG. Comparison of impression materials for direct multi-implant impressions. J Prosthet Dent. 2000; 83:32331.

3. Holst S, Blatz MB, Bergler M, Goellner M, Wichmann M. Influence of impression material and time on the 3-dimensional accuracy of implant impressions. Quintessence Int. 2007; 38: 67-73.

4. Lee H, So JS, Hochstedler JL, Ercoli C. The accuracy of implant impressions: a systematic review. J Prosthet Dent. 2008; 100:285-91

5. Del'Acqua MA, Chavez AM, Amaral ALC, Compagnoni MA, De Assis Mollo F. Comparison of impression techniques and materials for an implant supported prosthesis. Int J Oral Maxillofac Implants. 2010; 25:771-6.

6. Öngül D, Gökcen-Röhling B, Sermet B, Keskin H. A comparative analysis of the accuracy of different direct impression techniques for multiple implants. Aust Dent J. 2012; $57: 184-9$

7. Papaspyridakos P, Lal K, White GS, Weber HP, Gallucci GO. Effect of splinted and non-splinted impression techniques on the accuracy of fit of fixed implant prostheses in edentulous patients: a comparative study. Int J Oral Maxillofac Implants 2011; 26:1267-72.

8. Assunção WG, Filho HG, Zaniquelli O. Evaluation of transfer impressions for osseointegrated implants at various angulations. Implant Dent 2004; 13: 358-66. 
9. Conrad HJ, Pesun IJ, DeLong R, Hodges JS. Accuracy of two impression techniques with angulated implants. J Prosthet Dent 2007; 97:349-56.

10. Sorrentino R, Gherlone EF, Calesini G, Zarone F. Effect of implant angulation, connection length, and impression material on the dimensional accuracy of implant impressions: an in vitro comparative study. Clin Implant Dent Relat Res 2010; 12(suppl 1):e63-76.

11. Mpikos P, Kafantaris N, Tortopidis D, Galanis C, Kaisarlis G, Koidis P. The effect of impression technique and implant angulation on the impression accuracy of externaland internal connection implants. Int $\mathbf{J}$ Oral Maxillofac Implants 2012; 27:1422-8.

12. Carr AB. Comparison of impression techniques for a five implant mandibular model. International Journal of Oral \&Maxillofacial Implants 1991; 6:448-55.

13. Vojdani M, Torabi K, Ansarifard E. Accuracy of different impression materials in parallel and nonparallel implants. Dental research journal. 2015; 12(4):315.

14. Cehreli MC, Akça K. Impression techniques and misfitinduced strains on implant-supported superstructures: An in vitro study. Int J Periodontics Restorative Dent 2006; 26:379-85.

15. Enkling N, Bayer S, Jöhren P, Mericske-Stern R. Vinylsiloxanether: A new impression material. Clinical study of implant impressions with vinylsiloxanether versus polyether materials. Clin Implant Dent Relat Res 2012; 14:144-51.

16. Shetty S, Kamat G, Shetty R. Wettability changes in polyether impression materials subjected to immersion disinfection. Dent Res J (Isfahan) 2013; 10:539-44.

17. Christopher JB. Obtaining a high-quality impression with a new elastomeric material. J Inside Dent 2011; 7: 56-61.

18. Daoudi MF, Setchell DJ, Searson LJ. An evaluation of three implant level impression techniques for single tooth implant. Eur J Prosthodont Restor Dent. 2004; 12(1):9-14.

19. Mostafa TM, Elgendy MN, Kashef NA, Halim MM. Evaluation of the precision of three implant transfer impression techniques using two elastomeric impression materials. Int J Prosthodont 2010; 23:525-8.
20. Vigolo P, Majzoub Z, Cordioli G. Evaluation of the accuracy of three techniques used for multiple implant abutment impressions. Journal of Prosthetic Dentistry 2003; 89:186-92.

21. Kim S, Nicholls JI, Han CH, Lee KW. Displacement of implant components from impressions to definitive casts. Int J Oral Maxillofac Implants. 2006; 21:747-755.

22. Lee H, Ercoli C, Funkenbusch PD, Feng C. Effect of subgingival depth of implant placement on the dimensional accuracy of the implant impression: An in vitro study. J Prosthet Dent. 2008; 99:107-113.

23. Aguilar ML, Elias A, Vizcarrondo CE, Psoter WJ. Analysis of three-dimensional distortion of two impression materials in the transfer of dental implants. J Prosthet Dent 2010; 103:202-9.

24. Stober T, Johnson GH, Schmitter M. Accuracy of the newly formulated vinyl siloxanether elastomeric impression material. J Prosthet Dent 2010; 103:228-39.

25. Shankar YR, Sahoo S, Krishna MH, Kumar PS, Kumar TS, Narula S. Accuracy of implant impressions using various impression techniques and impression materials. Journal of Dental Implants. 2016; 6(1):29.

26. Prithviraj DR, Pujari M, Garg P, Shruthi DP. Accuracy of implant impression obtained from different impression materials and techniques: Review. J Clin Exp Dent 2011; 3:106-11.

27. Pandey A, Mehtra A. Comparative study of dimensional stability and accuracy of various elastomeric materials. IOSR J Dent Med Sci 2014; 13:40-5.

28. Misch C, editor. Available bone and dental treatment plans. In Contemporary Implant Dentistry. 3rd ed. St. Louis: Mosby; 1993. p. e184.

29. Stimmelmayr M, Güth JF, Erdelt K, Happe A, Schlee M, Beuer F. Clinical study evaluating the discrepancy of two different impression techniques of four implants in an edentulous jaw. Clin Oral Investig 2013;17:1929-35.

30. Akalin ZF, Ozkan YK, Ekerim A. Effects of implant angulation, impression material, and variation in arch curvature width on implant transfer model accuracy. Int J Oral Maxillofac Implants 2013;28:149-57. 